\title{
Study on the Correlation between Tinnitus and Depression in Elderly Depressive Patients
}

\author{
Ding Yuntong ${ }^{1}$, Xu Yayun ${ }^{3}$, Cheng L long ${ }^{3}$, Yan Fanfan ${ }^{3}$, Wang Jingliang ${ }^{3}$ and Zhang Xulai*2 \\ ${ }^{1}$ Department of Medical Psychology, China \\ ${ }^{2}$ Clinical School of Mental Health, China \\ ${ }^{3}$ The Fourth People's Hospital of Hefei, China
}

*Corresponding author: Zhang Xulai, Clinical School of Mental Health, Hefei 230022, China

\section{ARTICLE INFO}

Received: 幽 October 05, 2019

Published: 幽 October 11, 2019

Citation: Ding Yuntong, Xu Yayun, Cheng L long, Yan Fanfan, Wang Jingliang, Zhang Xulai. Study on the Correlation between Tinnitus and Depression in Elderly Depressive Patients. Biomed J Sci \& Tech Res 21(5)-2019. BJSTR. MS.ID.003674.

Keywords: Senile Depression; Tinnitus; Hamilton Depression Scale; Tinnitus Disability Scale

Abbreviations: THI: Tinnitus Handicap Inventory; HAMD: Hamilton Depression Scale; BNF: Brain Neurotrophic Factor

\section{ABSTRACT}

Objective: To investigate the occurrence of tinnitus in elderly depressive patients and the correlation between the degree of depression and disability caused by tinnitus.

Methods: A total of 126 patients with DSM-V depression in geriatric ward and outpatient department of Hefei Fourth People's Hospital from May 2019 to August 2019 were collected. Patients with depression accompanied by tinnitus were screened according to the standardization of tinnitus. According to the occurrence of tinnitus, patients were divided into depressive tinnitus group and depressive non-tinnitus group. The tinnitus disability scale (THI) and Hamilton Depression Scale (HAMD) were used to evaluate the degree of tinnitus disability and depression in patients. The difference of the degree of depression between the two groups was compared, and the correlation between the degree of depression and tinnitus disability was evaluated.

Results: Tinnitus occurred in 34 out of 126 elderly depressive patients (27\%). Compared with non-tinnitus depression group, the total score of HAMD $(t=3.727, p<0.01)$ and factor score of somatization $(\mathrm{t}=3.33, \mathrm{p}=0.01)$, cognitive impairment $(\mathrm{t}=2.961$, $\mathrm{p}=0.04)$, sleep disorder $(\mathrm{t}=4.04, \mathrm{p}<0.01)$ and despair $(\mathrm{t}=4.604, \mathrm{p}<0.01)$ in tinnitus depression group were significantly higher than those in non-tinnitus depression group. Spearman correlation analysis showed that there was a positive correlation between the disability grade of tinnitus and the degree of depression $(\mathrm{r}=0.377, \mathrm{P}=0.033)$.Pearson correlation analysis showed that the total score of THI was positively correlated with the total score of HAMD ( $\mathrm{r}=0.442, \mathrm{P}=0.011)$. Further analysis of the correlation between the total score of THI and the factors of HAMD showed that the total score of THI was positively correlated with cognitive impairment of factor score $(\mathrm{r}=0.434, \mathrm{P}=0.010)$, but not with body weight, block, sleep disorder, despair, day and night changes and somatization.

Conclusion: The incidence of tinnitus is higher in elderly depressive patients, and there is a certain correlation between the degree of tinnitus disability and the degree of depression.

\section{Introduction}

Depression is a common disease. According to the World Health Organization report, there are about 350 million people with depression worldwide. The number of suicide deaths due to depression is as high as 1 million per year. It is expected that by 2030, depression will become the world's first high-burden disease $[1,2]$. Geriatric depression is an important medical phenomenon in the aging society of the population, and it is a common cause of disability in the elderly and a decline in the quality of life in later life [3-5]. Elderly patients with depression are often accompanied by a variety of physical discomfort, such as tinnitus, which brings many difficulties to treatment. Tinnitus is an abnormal sound sensation caused by auditory dysfunction in the absence of external sound and sound stimulation [6]. About a quarter of the population has had a tinnitus experience [7]. 
The incidence of tinnitus increases rapidly with age, with an incidence of more than $10 \%$ among the elderly over 65 years of age, often accompanied by hearing loss [8]. Due to the obvious subjective response, patients with tinnitus often present with anxiety, depression and sleep disorders, which seriously affect normal life and work [9], and even lead to suicidal tendencies $[9,10]$. Studies have shown that tinnitus has a certain relationship with emotional disorders such as anxiety and depression [11], especially severe tinnitus, which is considered to be positively correlated with the degree of depression [12]. However, most of the current researches are based on the general population, and few patients with depression diagnosed as psychiatric patients are studied and analyzed. Therefore, this study intends to select elderly patients with depression to investigate the population, in order to explore the relationship between depressive symptoms and tinnitus in elderly patients with depression.

\section{Object / Material and Methods}

\section{Object / Material}

Elderly patients with depression who were admitted to the Geriatrics Department of Anhui Mental Health Center from May to August 2019 were included in the criteria:

a. Met the diagnostic criteria for DSM-V depression;

b. Older than 60 years old;

c. I and my guardian agree to participate in this study.

Exclusion Criteria:

a. Severe cardiovascular and cerebrovascular diseases, patients who cannot cooperate with physical examination and questionnaire;

b. Patients with otitis media, Meniere's disease, transient cerebral ischemia (TIA), and tinnitus after ear trauma

c. Combine the deafness or ear to hear other sounds such as vascular beat sounds.

Collect general patient information and sociodemographic data, including gender, age, working status, years of education, marital status, smoking behavior, age at onset of depression, total duration of illness, total number of hospitalizations, and drug use. The study was reviewed and approved by the Medical Ethics Committee of the Fourth People's Hospital of Hefei. All subjects or their guardians understood the research content and signed written informed consent. A total of 126 elderly patients with depression were enrolled, aged (68.61 \pm 6.124$)$ years, including 35 males $(28 \%)$ and 91 females (72\%).

\section{Methods}

General Information: The history of the collection is detailed to ask the patient's age, gender, height, weight, marital status, religious beliefs, years of schooling, time of onset of depression, duration of illness, number of hospitalizations, treatment status and family history, past medical history (focus on whether there is Mental illness, ear disease, high blood pressure, diabetes and cardiovascular and cerebrovascular diseases and smoking and drinking history).

Collection of Tinnitus and Disability Data: Use tinnitus standardization to determine whether there is tinnitus. The standardization question is: "Is there or noise in one or both ears in your mind (such as ringing or squeaking, Beep), a duration of more than five minutes?

A) Yes, most of the time has or has been;

B) Yes, often;

C) Yes, sometimes;

D) No.

The patient who answered "Yes" was determined to have tinnitus, and the patient who answered "No" was determined not to have tinnitus [13-16]. According to the results of standardization of tinnitus, the subjects were divided into two groups: depression with tinnitus group and depression without tinnitus group. Tinnitus severity assessment: The degree of disability of the patient's tinnitus was assessed using the internationally accepted Tinnitus handicap inventory (THI). The scale has a total of 25 questions, asking patients on a case-by-case basis, and answering "yes", "sometimes" and "none" according to the patient's own situation. If you select "Yes", it is recorded as 4 points, "sometimes" is recorded as 2 points, "None" is recorded as 0 points, and the highest is 100 points. According to the scale score, the tinnitus disability is divided into four levels [17], the first level: 0-16 points, no disability; the second level: 18-36 points, mild disability; the third level: $38-56$ points, medium Degree of disability; Level 4: 58-100 points, severe disability.

The Hamilton Depression Scale (HAMD): The Hamilton depression scale (HAMD) compiled by Hamilton in 1960, is currently the most commonly used gauge for assessing depression status. The study used 24 versions and was evaluated by the same psychiatrist based on the patient's symptoms. The assessment included total scores and factor scores, including factors such as somatization, weight, cognitive impairment, day and night changes, block, Sleep disorders and sense of despair.

Statistical methods were Analyzed by SPSS 17.0 Software: The measurement data were expressed as $\mathrm{x} \pm \mathrm{s}$. The $\mathrm{t}$ test was used to compare the two groups of measurement data. The chi-square test was used to compare the two groups of data. The Pearson correlation analysis was used to evaluate the THI scale score and Hamilton. Correlation of depression scale factors, Spearman correlation analysis was used to assess the correlation between tinnitus disability grade and Hamilton depression score. The difference was statistically significant at $\mathrm{P}<0.05$.

\section{Results}

(1) Depression of tinnitus group and depression non- tinnitus group. General data. Gender, age, BMI, marriage, education level, smoking status, drinking status, history of hypertension, history of 
diabetes, history of cerebral infarction, age of onset of depression There was no significant difference in the difference ( $p>0.05)$. The number of hospitalizations in the depression and tinnitus group was significantly higher than that in the depression-free non-Tining group. The difference was statistically significant $(\mathrm{t}=2.138, \mathrm{p}=0.035)$, as shown in Table 1.

Table 1: Comparison of general data and clinical data between depression and tinnitus group.

\begin{tabular}{|c|c|c|c|c|}
\hline Item & Tinnitus Group & Non-Tining Group & tor $\chi^{2}$ & $p$ value \\
\hline Gender & & & -0.4 & 0.842 \\
\hline Male & 9 & 26 & & \\
\hline Female & 25 & 66 & & \\
\hline Age & $68.18 \pm 4.522$ & $68.62 \pm 6.879$ & -0.419 & 0.676 \\
\hline BMI kg $/ \mathrm{m}^{2}$ ) & $21.6730 \pm 2.757$ & $22.5064 \pm 2.988$ & -1.367 & 0.174 \\
\hline Education (year) & $4.53 \pm 4.844$ & $4.18 \pm 3.983$ & 0.406 & 0.685 \\
\hline Marriage & & & -0.689 & 0.406 \\
\hline Married & 31 & 89 & & \\
\hline Unmarried & 0 & 0 & & \\
\hline Widowed & 3 & 3 & & \\
\hline Divorced & 0 & 0 & & \\
\hline Faith & & & -1.591 & 0.207 \\
\hline Yes & 5 & 23 & & \\
\hline No & 59 & 68 & & \\
\hline Income (yuan) & & & -1.379 & 0.502 \\
\hline Less than 1500 & 19 & 47 & & \\
\hline $1500-4500$ & 13 & 42 & & \\
\hline Greater than 4500 & 1 & 2 & & \\
\hline Previous drinking & & & -3.414 & 0.445 \\
\hline No & 3 & 73 & & \\
\hline Light & 0 & 3 & & \\
\hline In & 2 & 2 & & \\
\hline Weight & 2 & 11 & & \\
\hline Serious & 0 & 3 & & \\
\hline Smoking & & & -0.26 & 0.823 \\
\hline Never & 28 & 72 & & \\
\hline Smoking & 3 & 10 & & \\
\hline Quit Smoking & 3 & 10 & & \\
\hline Hypertension & & & -2.929 & 0.087 \\
\hline Yes & 8 & 9 & & \\
\hline No & 26 & 83 & & \\
\hline Brain Infarction & & & -0.001 & 0.982 \\
\hline Yes & 3 & 8 & & \\
\hline No & 31 & 84 & & \\
\hline Coronary Heart Disease & & & 0.374 & 0.541 \\
\hline Yes & 1 & 5 & & \\
\hline No & 33 & 87 & & \\
\hline Depression First Age & $61.85 \pm 9.471$ & $61.11 \pm 14.062$ & 2.85 & 0.776 \\
\hline First Recurrence & & & -1.201 & 0.273 \\
\hline Starting & 8 & 31 & & \\
\hline Relapse & 26 & 61 & & \\
\hline Psychotic Symptoms & & & -0.116 & 0.734 \\
\hline Accompanied by & 2 & 7 & & \\
\hline Not Accompanied & 32 & 85 & & \\
\hline Number of hospitalizations (times) & 2.15 & 1.38 & 2.138 & 0.035 \\
\hline
\end{tabular}


(2) Depression in the tinnitus group and depression in the non-Tining group Hamilton score compared with the depression non-sounding group, depression with tinnitus group HAMD total score $(\mathrm{t}=3.727, \mathrm{p}<0.01)$ and factor scored somatization $(\mathrm{t}=3.33$, $\mathrm{p}=0.01)$, cognitive impairment $(\mathrm{t}=2.961, \mathrm{p}=0.04)$, sleep disturbance $(\mathrm{t}=4.04, \mathrm{p}<0.01)$ and sense of despair $(\mathrm{t}=4.604, \mathrm{p}<0.01)$ were higher than non-tinnitus group, the difference Statistically significant, see Table 2.

Table 2: Comparison of Hamilton Depression Scale scores between tinnitus group and non- tinnitus group in elderly patients with depression.

\begin{tabular}{|c|c|c|c|c|}
\hline Hamilton Depression Scale & Non-sounding group & Tinnitus group & t & $\mathbf{p}$ \\
\hline Somatization & $7.93 \pm 2.782$ & $9.74 \pm 2.391$ & -3.33 & 0.01 \\
\hline Cognitive Impairment & $2.89 \pm 2.255$ & $3.85 \pm 1.306$ & -2.961 & 0.04 \\
\hline Body Weight & $1.14 \pm 0.884$ & $1.09 \pm 0.933$ & 0.295 & 0.769 \\
\hline Block & $5.24 \pm 2.24$ & $5.97 \pm 1.977$ & -1.677 & 0.096 \\
\hline Sleep Disorder & $3.97 \pm 1.930$ & $5.15 \pm 1.234$ & -4.04 & $<0.01$ \\
\hline Day and Night Changes & $0.83 \pm 0.460$ & $0.71 \pm 0.524$ & 1.254 & 0.212 \\
\hline Despair & $4.60 \pm 2.209$ & $6.56 \pm 1.862$ & -4.604 & $<0.01$ \\
\hline Total Score & $24.73 \pm 8.493$ & $30.76 \pm 6.769$ & -3.727 & $<0.01$ \\
\hline
\end{tabular}

(3) Correlation analysis between tinnitus disability grade and depression degree the results of Spearman correlation analysis showed a positive correlation between tinnitus disability grade and depression degree ( $r=0.377, \mathrm{p}=0.033)$, see Table 3 .

Table 3: Correlation analysis between tinnitus disability grade and Hamilton depression total score.

\begin{tabular}{|c|c|c|}
\hline Tinnitus Level & Mild to Moderate Depression & Serious \\
\hline No Disability & 6 & 1 \\
\hline Mild Disability & 11 & 5 \\
\hline Moderate Disability & 1 & 3 \\
\hline Severe Disability & 2 & 5 \\
\hline
\end{tabular}

(4) Correlation analysis between THI total score and Hamilton Depression Scale total score and each factor Pearson correlation analysis showed that THI total score was positively correlated with HAMD total score $(r=0.442, p=0.011)$. Further analysis of the correlation between the THI total score and each factor showed that the THI total score was positively correlated with the cognitive impairment factor score ( $\mathrm{r}=0.434, \mathrm{p}=0.010)$, but with weight, block, sleep disorder, and sense of despair. There is no obvious correlation between day and night changes and somatization, see Table 4 .

Table 4: Correlation between THI scale score and Hamilton depression scale factor score.

\begin{tabular}{|c|c|c|}
\hline \multicolumn{2}{|c|}{$\mathbf{r}$} & $\mathbf{p}$ \\
\hline Total score & 0.442 & 0.011 \\
\hline Cognitive Impairment & 0.413 & 0.015 \\
\hline Body Weight & 0.037 & 0.838 \\
\hline Block & 0.032 & 0.858 \\
\hline Sleep Disorder & 0.04 & 0.822 \\
\hline Despair & 0.125 & 0.48 \\
\hline Day and Night Changes & 0.057 & 0.748 \\
\hline Somatization & -0.103 & 0.563 \\
\hline \multicolumn{2}{|c|}{ (More than one line? Background removed) } \\
\hline
\end{tabular}

\section{Discussion}

This study investigated 126 elderly patients with depression who met the DSM-V diagnostic criteria at the Fourth People's Hospital of Hefei and found that the incidence of tinnitus reached 27.0\%. Compared with the depression-free non-Tining group, the HAMD total score and factor scores of somatosensory, cognitive impairments, sleep disturbance, and despair were significantly increased in the depression tinnitus group. The results of the Spearman correlation analysis showed a positive correlation between the level of tinnitus disability and the degree of depression. Pearson correlation analysis showed that THI total score was positively correlated with HAMD total score and cognitive impairment factor score, suggesting that there may be a certain correlation between the level of tinnitus disability and the degree of depression in elderly patients with depression. Tinnitus is a common symptom of otolaryngology, and it is also common in patients with depression and anxiety disorders.

Psychiatrists are also beginning to pay attention to the physical symptoms of patients with mental disorders. An epidemiological survey in Australia showed that tinnitus had occurred in 30.3\% of the elderly (age 55-99) [13]. This is consistent with the results of this study. This study found that the incidence of tinnitus in the elderly patients with depression reached $27.0 \%$, suggesting that clinicians should pay close attention to the occurrence of tinnitus in 
elderly patients while treating depression in elderly patients. This study found that the total score of HAMD in the depression tinnitus group was significantly higher than that in the depression nontinnitus group, indicating that tinnitus may aggravate depressionrelated symptoms in elderly patients with depression. The specific mechanism is not clear. Studies have shown that over-activated cochlear dorsal nucleus in tinnitus may control emotional response by projecting into areas such as the blue spot, reticular structure, and nucleus of the nucleus [14]. Studies of neuroendocrine function have shown that tinnitus may induce an exacerbation of depressive symptoms by modulating the function of the HPA axis [15].

Animal studies have found that hippocampal damage in tinnitus model rats may be associated with depression performance [16] The above mechanism studies and the results of this paper suggest that tinnitus may be a potential indicator for judging the severity of depression in the elderly, that is, when the patient has tinnitus symptoms, it may indicate more serious depressive symptoms. Reports at home and abroad suggest a link between tinnitus and depression. Tinnitus can induce and aggravate the occurrence of depression [12,17], while adverse emotions such as depression and anxiety can also cause or aggravate tinnitus [18]. Consistent with previous studies $[13,19]$, this study found a positive correlation between the level of tinnitus disability and the degree of depression. Analysis of the reasons may be related to the mechanism of tinnitus and depression. The current medical opinion is that tinnitus originates from the cochlea and forms in the center. The occurrence and development of tinnitus are closely related to the central nervous system. Previous studies have suggested that the occurrence of tinnitus is likely to be related to serotonin (5-HT) and glutamic acid in the central nervous system. Dysfunction and disorders of limbic system function [20-22], while the limbic system and neurotransmitters such as 5-HT and glutamate play an important role in the pathogenesis of depression [23].

In addition, the "gating" system of tinnitus [24] is thought to be a key factor in the comorbid mechanism of tinnitus and depression. On the one hand, the weakening and destruction of the system's function is related to tinnitus symptoms [25]. On the other hand, the system is susceptible to emotions, and negative emotions can reduce its function [26]. Human genomics studies suggest that brain neurotrophic factor (BNF) gene mutations may be a common susceptibility to both diseases [27]. In this study, Pearson correlation analysis showed a positive correlation between THI total score and cognitive impairment factor scores. Similarly, studies have shown that cognitive decline such as "short-term memory", "attention ability" and "directed ability" are the main clinical features of cognitive impairment in patients with chronic subjective tinnitus [28]. In view of the close relationship between the ventromedial cortex of the prefrontal cortex and cognitive functions such as attention and memory [29], Leaver et al. believe that the abnormality of the ventromedial cortex in the prefrontal cortex of tinnitus patients may be the cause of cognitive impairment in patients [30].
The Main Disadvantages of this Study are:

1) This study is a cross-sectional study. It can only analyze the relationship between tinnitus and depression and clinical features. It is impossible to infer the causal relationship between depression and tinnitus, and it is necessary to continue the prospect of strict design. Sexual research;

2) Enrolled patients were from the Fourth People's Hospital of Hefei City, with limited representation, especially 34 patients with tinnitus, more sample, a larger range of studies will help reduce research bias;

3) Depression and Tinnitus is closely related, but the hearing indicators of tinnitus patients are not detected in this study, so further research is needed to explore.

4) This study is only a current survey, and the exact relationship between tinnitus and depression needs further explanation from the etiology. The next work will further explain the interaction between the nervous system structure and EEG to more Good guidance for clinical work.

In short, tinnitus is a common clinical symptom. Psychiatric patients, especially those with depression in the elderly, have a high incidence. It seriously affects the physical and mental health of patients. Because the pathogenesis of tinnitus is still unclear, the lack of treatment methods and evaluation criteria is difficult to cause symptoms. Relieve, bring a serious psychological burden to patients, then further explore the relationship between the two to help to better understand the pathogenesis of depression, while in the clinical discovery of depression with tinnitus patients, while paying attention to depression, the concern for tinnitus cannot be ignored.

\section{Acknowledgement}

Key research and development project of Anhui provincial science and technology department (201904a0702009) (consistent with Chinese).

\section{References}

1. (2008) Organization WH, The global burden of disease: 2004 update.

2. Yue Xianning, Liu Zhengfu, Ye Lanxian (2016) Progress in the treatment of refractory depression. Chinese Journal of Behavioral Medicine and Brain Science 25(3): 280-284.

3. Zhang Y, Yeager VA, Hou S (2018) The Impact of Community-Based Supports and Services on Quality of Life Among the Elderly in China: A Longitudinal Study. J Appl Gerontol 37(10): 1244-1269.

4. Beekman AT, Copeland JR, Prince MJ (1999) Review of community prevalence of depression in later life. Br J Psychiatry 174: 307-311.

5. Bishwajit G, O'Leary DP, Ghosh S, Yaya S, Shangfeng T, et al. (2017) Physical inactivity and self-reported depression among middle- and older-aged population in South Asia: World health survey. BMC Geriatr 17(1): 100.

6. David J (2005) Tinnitus and its American diagnosis and treatment guidelines. Chinese Journal of Hearing and Speech Rehabilitation 1: 5861.

7. Shargorodsky J, Curhan GC, Farwell WR (2010) Prevalence and characteristics of tinnitus among US adults. Am J Med 123(8): 711-718. 
8. Levine RA, Oron Y (2015) Tinnitus. Handb Clin Neurol 129: 409-431.

9. Adoga AA, Kokong DD, Nimkur TL, Okwori ET (2015) The impact of tinnitus on adult Nigerians: health related Quality of Life assessment of sufferers using the Hospital Anxiety and Depression Scale (HADS) and the RAND-36 item health survey 1.0 questionnaire. Int Tinnitus J 19(2): 26-32.

10. Aazh H, Landgrebe M, Danesh AA (2019) Parental Mental Illness in Childhood as a Risk Factor for Suicidal and Self-Harm Ideations in Adults Seeking Help for Tinnitus and/or Hyperacusis. Am J Audiol 28(3): $527-$ 533.

11. Ziai K, Moshtaghi O, Mahboubi H, Hamid R Djalilian (2017) Tinnitus Patients Suffering from Anxiety and Depression: A Review. Int Tinnitus J 21(1): 68-73.

12. Kehrle HM, Sampaio AL, Granjeiro RC, De Oliveira TS, Oliveira CA (2016) Tinnitus Annoyance in Normal-Hearing Individuals: Correlation With Depression and Anxiety. Ann Otol Rhinol Laryngol 125(3): 185-194.

13. Sindhusake D, Mitchell P, Newall P, Golding M, Rochtchina E, et al. (2003) Prevalence and characteristics of tinnitus in older adults: the Blue Mountains Hearing Study. Int J Audiol 42(5): 289-294.

14. Langguth B, Landgrebe M, Kleinjung T, Sand GP, Hajak G (2011) Tinnitus and depression. World J Biol Psychiatry 12(7): 489-500.

15. Birgit M, Heidemarie H, Heidi O, Agnieszka J Szczepek (2012) Stress and tinnitus-from bedside to bench and back. Front Syst Neurosci 6: 47.

16. Eggermont JJ, Roberts LE (2015) Tinnitus: animal models and findings in humans. Cell Tissue Res 361(1): 311-336.

17. Mc Cormack A, Edmondson Jones M, Fortnum H, Dawes PD, Middleton $\mathrm{H}$ et al. (2015) Investigating the association between tinnitus severity and symptoms of depression and anxiety, while controlling for neuroticism, in a large middle-aged UK population. Int J Audiol 54(9): 599-604.

18. Bhatt JM, Bhattacharyya N, Lin HW (2017) Relationships between tinnitus and the prevalence of anxiety and depression. Laryngoscope 127(2): 466-469.

19. Li Wei, Wang Mingxi, Zhou Wei (2019) Study on anxiety and depression status and related factors in patients with idiopathic tinnitus. Journal of Clinical Otorhinolaryngology Head and Neck Surgery 33(5): 37-42.

ISSN: 2574-1241

DOI: 10.26717/BJSTR.2019.21.003674

Zhang Xulai. Biomed J Sci \& Tech Res

This work is licensed under Creative

Commons Attribution 4.0 License

Submission Link: https://biomedres.us/submit-manuscript.php
20. Chen YC, W Xia, Chen H, Feng Y, Xu JJ, et al. (2017) Tinnitus distress is linked to enhanced resting-state functional connectivity from the limbic system to the auditory cortex. Hum Brain Mapp 38(5): 2384-2397.

21. Besteher B, Gaser C, Ivansic D, Orlando Guntinas Lichius, Christian Dobel, et al. (2019) Chronic tinnitus and the limbic system: Reappraising brain structural effects of distress and affective symptoms. Neuroimage Clin 24: 101976.

22. Sun W, Lu J, Stolzberg D, Gray L, Deng A, et al. (2009) Salicylate increases the gain of the central auditory system. Neuroscience 159(1): 325-334.

23. Ménard C, Hodes GE, Russo SJ (2016) Pathogenesis of depression: Insights from human and rodent studies. Neuroscience 321: 138-162.

24. Rauschecker JP, May ES, Maudoux A, Ploner M (2015) Frontostriatal Gating of Tinnitus and Chronic Pain. Trends Cogn Sci 19(10): 567-578.

25. Lai Renzhen, Ma Xin (2017) Switch to Hearing Loss and Tinnitus--A Control Gate. Journal of Clinical Otorhinolaryngology Head and Neck Surgery 31(7): 6-8.

26. PANG Ying, ZENG Ji Hong, KANG Hou Xi (2018) Research progress in the pathogenesis of tinnitus. International Journal of Otorhinolaryngology Head and Neck Surgery 42(6): 330-334.

27. Coskunoglu A, Orenay Boyacioglu S, Deveci A, Bayam M, Onur E, et al (2017) Evidence of associations between brain-derived neurotrophic factor (BDNF) serum levels and gene polymorphisms with tinnitus. Noise Health19(88): 140-148.

28. Zhang Jianping, Cao Tingting, Ren Bingqi (2018) Characteristics of cognitive impairment of chronic subjective tinnitus. Journal of Apoplexy and Nervous Diseases 35(12): 57-60.

29. Lee YJ, Bae SJ, Lee SH, Lee JJ, Lee KY, et al. (2007) Evaluation of white matter structures in patients with tinnitus using diffusion tensor imaging. J Clin Neurosci 14(6): 515-519.

30. Leaver AM, Seydell Greenwald A, Turesky TK, Susan Morgan, Hung J Kim et al. (2012) Cortico-limbic morphology separates tinnitus from tinnitus distress. Front Syst Neurosci 6: 21.

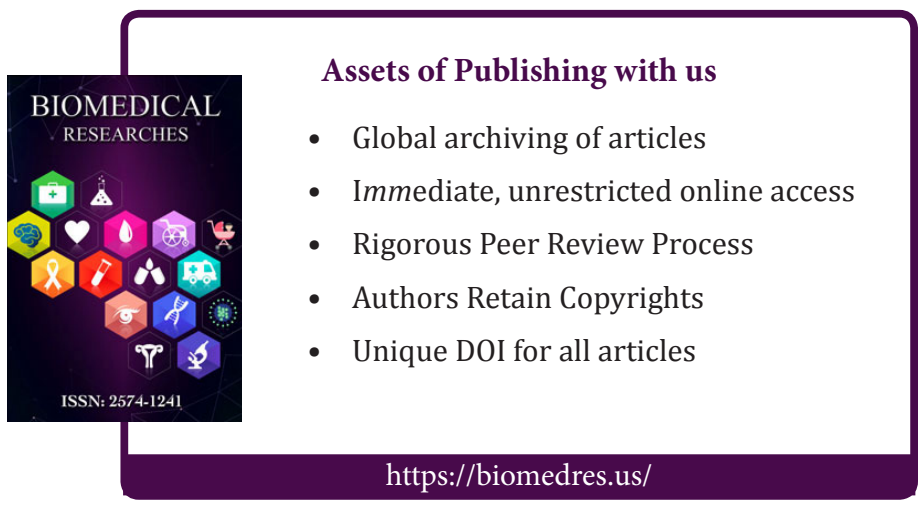

